\title{
DESENVOLVIMENTO DE UM MODELO PARA ESCOAMENTO DE ÓLEOS PARAFÍNICOS
}

\author{
M. L. AlCANTARA ${ }^{1}$, G. B. TARANTINO ${ }^{1}$, S. MATTEDI $^{1}$, L. M. N. GOES ${ }^{1}$, L. C. \\ L. SANTOS ${ }^{1}$ e C. A. PIRES ${ }^{1}$ \\ ${ }^{1}$ Programa de Pós-Graduação em Engenharia Química (PPEQ), Universidade Federal \\ da Bahia (UFBA) \\ E-mail para contato: cap@ufba.br
}

\begin{abstract}
RESUMO - A deposição de parafinas é um dos problemas na produção e transporte do petróleo. Compreender o fenômeno da precipitação de parafina é o primeiro passo para garantir a produção e escoamento de petróleo com segurança e economia. Este trabalho tem como objetivo desenvolver um modelo que consiga estimar a perda de carga em escoamento de óleos com alta concentração de nparafinas. Foram realizados experimentos com óleos parafínicos extraídos de diversos poços. Alguns parâmetros do modelo foram medidos experimentalmente. A temperatura de aparecimento de cristais (TIAC) foi estimada através de microcalorimetria e o comportamento da viscosidade do fluido por viscosimetria. Neste trabalho foi desenvolvido um modelo semi-empírico capaz de estimar a perda de carga do escoamento de óleos parafínicos em diferentes condições de teor de água, vazão e temperatura ambiente. Os resultados obtidos mostraram que o modelo representa físico-quimicamente o fenômeno estudado.
\end{abstract}

\section{INTRODUÇÃO}

Com o aumento da demanda mundial de petróleo e seus derivados, cada vez mais é necessário o transporte e processamento destes fluidos. A depender das condições de trabalho, um problema comum que pode ocorrer é a precipitação de ceras e hidratos. Um dos principais desafios da engenharia de produção é evitar a precipitação destes componentes nos poços, dutos e equipamentos de superfície.

O foco deste trabalho esta na precipitação de parafinas, que é um dos principais problemas na produção e armazenamento de petróleo. Este fenômeno causa prejuízos consideráveis a indústria petrolífera todos os anos, resultando em elevados gastos com manutenção de instrumentos e energia. A precipitação de ceras pode causar danos irreparáveis a equipamentos, operação em condições de elevado risco e, em casos extremos, parada completa da produção (PAULY; DARIDON; COUTINHO, 2004).

Vários modelos de escoamento de fluidos já foram desenvolvidos até o momento. Os modelos de Hagen-Poiseuille e Darcy-Weisbach são alguns dos mais conhecidos e aplicados, principalmente, em escoamento de fluidos incompressíveis e newtonianos. Devido à natureza complexa dos escoamentos de petróleo, modelos mais específicos são necessários para prever o escoamento destes óleos. Alguns destes modelos são os aplicados por Abduvayt et al. 
(2002) e Hasan et al. (2010), empregados no escoamento bifásico, vertical ou horizontal sem a precipitação de parafinas. Estudos do fenômeno de crstalização e precipitação de n-parafinas têm sido realizados em diversas áreas e com diversas aplicações, o impacto deste fenômeno no escoamento de petróleo foi estudado por Coto et al. (2010), Santos et al. (2004) e Shagapov et. al. (2004). Entretanto, na literatura pesquisada até o momento, não foi encontrado nenhum trabalho capaz de prever o comportamento e quantificar o efeito da perda de carga ocasionada pela precipitação de n-parafina em escoamento unifásico.

Neste contexto, o objetivo deste trabalho é desenvolver um modelo fenomenológico capaz de prever o comportamento da perda de carga no escoamento horizontal e unifásico de petróleo com alta concentração de n-parafinas.

\section{MATERIAIS E MÉTODOS}

\subsection{Procedimento Experimental}

Utilizando uma unidade experimental foi possível efetuar experimentos com óleos escoando a diferentes vazões (Q), teores de água (estimado através do Basic Sediment and Water, BSW) e em diferentes temperaturas (T), como pode ser observado na Tabela 1.

Tabela 1 - Variáveis de Processo

\begin{tabular}{lcccccccc}
\hline Experimento & EXP1 & EXP2 & EXP3 & EXP4 & EXP5 & EXP6 & EXP7 & EXP8 \\
\hline BSW $(\%)$ & 5 & 5 & 5 & 5 & 35 & 35 & 35 & 35 \\
Q $(\mathrm{mL} / \mathrm{min})$ & 150 & 150 & 200 & 200 & 150 & 150 & 200 & 200 \\
$\mathrm{~T}\left({ }^{\circ} \mathrm{C}\right)$ & 20 & 25 & 20 & 25 & 20 & 25 & 20 & 25 \\
\hline
\end{tabular}

Unidade experimental: A unidade experimental utilizada nos ensaios foi o WAX LOOP 208 da PSL Systemtechnik com banho criostático modelo P2-C50 e um software de controle, aquisição e análise de dados WL_Control. Este equipamento pode ser considerado como um simulador de escoamento de produção no qual a amostra é bombeada com temperatura e vazão controlada. A variação de pressão do óleo é obtida a partir de medição diferencial que é continuamente registrada em um diagrama de tempo de ensaio. Um esquema da unidade experimental pode ser observado na Figura 1.

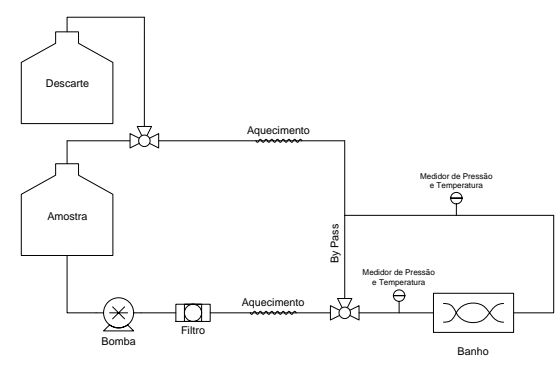

Figura 1 - Esquema simplificado da unidade experimental 
Viscosimetria e calorimetria: A viscosidade e a TIAC são duas das variáveis do modelo. Esses dois parâmetros foram estimadas experimentalmente, sendo a curva da viscosidade estimada através de medições constantes em um viscosímetro Brookfield. Já a TIAC foi obtida através de microcalorimetria, podendo ser observada através do único evento exotérmico.

\subsection{DESCRIÇÃO DO MODELO}

Observando o comportamento da perda de carga dos experimentos, é possível notar a existência de uma mudança no comportamento da perda de carga quando a temperatura do fluido se torna constante. Desta forma, foram desenvolvidos dois modelos para estimar o comportamento do escoamento em cada etapa, ambos baseados na Equação de HagenPoiseuille modificada.

Apesar de não representar os óleos parafínicos, a equação de Hagen Poiseuille (Equação 1) é um dos modelos de perda de carga mais utilizados pois consegue prever a perda de carga $(\Delta P)$ da maioria dos escoamentos. Sendo $C$ uma constante, $\mu$ a viscosidade, $L$ o comprimento da tubulação, $Q$ a vazão e $D$ o diâmetro.

$$
\Delta P=\frac{C \mu Q L}{D^{4}}
$$

Foram realizados experimentos com óleos não parafínicos e foi comprovado que a Equação 1 consegue prever o escoamento destes óleos, nas condições estudadas no presente trabalho e descritas na Tabela 1 , desde que ajustada a constante " $C$ " diferente para cada experimento. Entretanto, quando o escoamento é realizado em temperaturas inferiores a TIAC, a Equação 1 já não pode ser aplicada devido a mudança do comportamento do escoamento proveniente da precipitação de parte dos compostos do óleo, predominantemente parafinas. O efeito da deposição destes compostos foi estimado com base no efeito isolante térmico que a parafina causa ao precipitar nas paredes internas da tubulação (SANTOS et. al., 2004). Ou seja, quanto maior o número de carbonos de um composto do petróleo, maior é seu calor específico e consequentemente, menor a condutividade térmica do composto. Como as parafinas que primeiro depositam são as de cadeias longas, pode-se afirmar que o precipitado é composto por um dos piores condutores térmicos presentes no petróleo. Desta forma, considerou-se que todo o aumento da temperatura do fluido, observado nos experimentos, é resultado da formação de uma camada isolante de parafina na superfície interna da tubulação. Esta camada isolante reduz a perda de calor do fluido para o ambiente, o que resulta em um aumento na temperatura do fluido. Outra consequência da formação desta camada de precipitado é o aumento da perda de carga devido, dentre outros fatores, à redução do diâmetro efetivo da tubulação e ao aumento da rugosidade das paredes do sistema (SANTOS et. al., 2004).

Portanto, o modelo desenvolvido no presente trabalho quantifica a perda de carga ocasionada pela precipitação de parafinas através do efeito isolante térmico que a mesma provoca. O termo $f(T)$ (Equação 2) foi utilizado como conversor do fenômeno da precipitação de n-parafinas na perda de carga.

$$
f(T)_{i}=\left(1 / T s a i ́ d a_{\min }-1 / T s a i ́ d a_{i}\right)
$$


Onde Tsaída é o valor da temperatura do óleo na saída da unidade experimental, e o termo Tsaida $_{(\min )}$ representa a menor temperatura de saída observada durante todo o experimento. A temperatura de saída mínima foi considerada como a temperatura para um escoamento com precipitação mínima. Ou seja, qualquer aumento de temperatura acima desta temperatura mínima, pode ser resultado do isolamento térmico da camada de parafina depositada na superfície interna da tubulação.

A cristalização pode ser dividida em três etapas, a formação dos núcleos cristalinos, crescimento destes e formação de aglomerados. O modelo desenvolvido por este trabalho considera que, no período inicial, a perda de carga é proporcional à taxa de formação de núcleos cristalinos, observada na Equação 3. Desta forma, a Equação 4 foi desenvolvida com o objetivo de corrigir a Equação 1 para que esta se aplique aos óleos parafínicos. Observe que esta equação é a Equação 1 com um somatório de um termo semelhante à taxa de parafinação.

$$
\begin{aligned}
& I=\beta N \exp \left(-\Delta G^{*} / k_{B} T\right) \\
& \Delta P=\frac{C \mu Q L}{D^{4}}+\sum B e^{A f(T)}
\end{aligned}
$$

Nas Equações 3 e 4, $B$ e $A$ são constantes a serem estimadas, $\Delta G^{*}$ é a energia livre crítica correspondente ao do raio crítico, $N$ é o número de potenciais sítios de nucleação e $k_{B}$ representa a constante de Boltzmann.

A formação desta parede isolante é o fenômeno que rege o comportamento inicial dos experimentos. Entretanto, em períodos mais longos os cristais formados crescem e se aglomeram. Isto torna a camada de parafina mais espessa e, consequentemente, um melhor isolante térmico. Este fenômeno reduz o diferencial de temperatura entre o fluido e a superfície da tubulação, o que resulta em uma menor deposição de parafinas. Isto pode explicar a redução do crescimento da perda de carga dos experimentos e períodos maiores.

Outro fator que também acontece neste período é a redução da contribuição da deposição de cera no isolamento térmico do sistema. Isto faz com que, mesmo com deposição de parafinas na camada isolante, a temperatura de saída do fluido sofra um aumento de temperatura menos significativo. Isto pode justificar o crescimento da perda de carga durante este período, apesar da temperatura permanecer constante.

Com a finalidade de caracterizar o comportamento do segundo período dos experimentos, foi desenvolvida a Equação 5 cujo o objetivo é quantificar a contínua precipitação de parafinas durante este período. O modelo considera que quanto mais próxima à temperatura média do fluido estiver da sua respectiva TIAC maior será a influencia na precipitação, sendo o termo $f(T)$ calculado segundo a Equação 6.

$$
\begin{aligned}
& \Delta P=\frac{C \mu Q L}{D^{4}}+B \sum_{t=0}^{t_{n}} f(T) \\
& f(T)_{i}=1 /\left(\text { TIAC }-T_{(i)}\right)
\end{aligned}
$$

Outro fator que também deve ser considerado nos experimentos é o fenômeno do arraste do precipitado na superfície interna da tubulação. Segundo Santos (2004), a 
movimentação do fluido pode remover partículas das camadas superiores da parede de parafina. Como durante os experimentos a vazão é constante, quanto maior a espessura da parede de precipitado, menor será o diâmetro efetivo da tubulação e maior será a velocidade do fluido. Consequentemente, maior será a força de arraste exercida pelo fluido, nas partículas. A força de arraste foi estimada através da Equação de Newton (Equação 7),

$$
F_{\text {arrasto }}=\frac{1}{2} C_{a} \rho A v^{2}
$$

onde $v$ é a velocidade do fluido, $A$ é a área da projeção perpendicular da esfera, no sentido do escoamento, $\rho$ é a densidade do fluido e $C_{a}$ é a constante de arrasto.

A Equação de Newton calcula o arraste que um fluido faz sobre uma esfera. No presente trabalho, foi considerado que a força de arraste do fluido é diretamente proporcional ao volume de cera removida das paredes internas da tubulação (Equação 8).

$$
F_{\text {arrasto }}=C . V_{\text {cera arrastada }}
$$

Como dito anteriormente, a deposição de ceras nas paredes internas da tubulação ocasiona uma redução do diâmetro interno efetivo, no qual o fluido escoa. Como em todos os experimentos a vazão permanece constante, a redução do diâmetro efetivo da tubulação resulta em um aumento na velocidade do fluido. Desta forma, seria necessário saber o valor do volume de parafina que está precipitado na parede da tubulação para, então, estimar a redução do diâmetro interno efetivo.

O volume de parafina presente foi estimado então como sendo diretamente proporcional ao termo $f(T)$ (Equação 6) e a Equação 9 foi a expressão utilizada para este fim, onde $C_{t}$ é uma constante de ajuste.

$$
V_{\text {parafina }}=f(T) \cdot C_{t}
$$

Para a estimativa do raio interno efetivo, considerou-se que a precipitação da parafina ocorreu de forma uniforme na tubulação. Assim, formando um cilindro oco constituído cujos raios maior $(R)$ e menor ( $r$ ) são utilizados no cálculo do volume (Equação 10).

$$
V_{\text {parafina }}=\pi L R^{2}-\pi L r^{2}
$$

Onde, o raio interno efetivo $r$ e a velocidade do fluido $v$ podem ser estimados através das Equações 11 e 12 respectivamente.

$$
\begin{aligned}
& r^{2}=R^{2}-V_{\text {parafina }} / \pi L \\
& v=Q / \pi r^{2}
\end{aligned}
$$

Rearranjando as Equações 8, 9, 10, 11 e 12 pode-se obter a Equação 13.

$$
V_{\text {cera arrastada }}=\frac{C^{\prime} Q^{2}}{\left(R^{2}-C_{t} f(T) / \pi L\right)}
$$


De maneira semelhante ao ajuste efetuado na Equação 1, pode-se converter o volume de cera arrastada em $f^{\prime}(T)$ como observado na Equação 14.

$$
f^{\prime}(T)=V_{\text {cera arrastada }} / C_{t}
$$

Concluindo, a perda de carga para óleos parafínicos pode ser representada pela Equação 15, para longos períodos de escoamento.

$$
\Delta P=\frac{C \mu Q L}{D^{4}}+A_{1} \sum_{t=0}^{t_{n}}\left(f(T)-f^{\prime}(T)\right)
$$

\section{RESULTADOS E DISCUSSÕES}

Após os primeiros testes do modelo, os resultados mostram que havia muitas oscilações na temperatura de entrada do fluido na unidade experimental devido às limitações de um controle manual. Uma das formas de se tentar minimizar os erros operacionais é a linearização dos dados. Este procedimento foi efetuado apenas para as variáveis de temperatura no período inicial dos experimentos. Para as temperaturas de entrada, foi aplicada uma média aritmética, visto que esta variável apresenta um comportamento aproximadamente constante durante todo o experimento. Tanto temperatura de saída do fluido quanto a perda de carga estimada pelo modelo, medida experimentalmente e estimada para o escoamento de um óleo não parafínico (baixa fração de parafinas) podem ser observados nas Figuras 3 e 4.

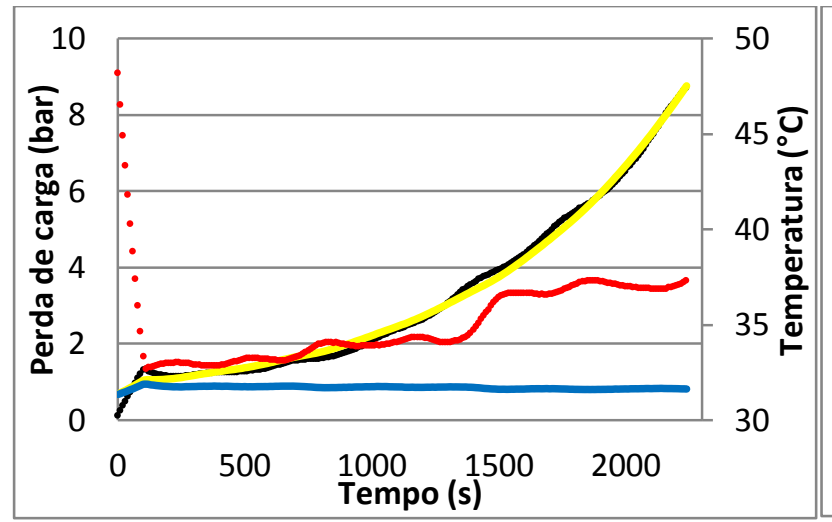

(a)

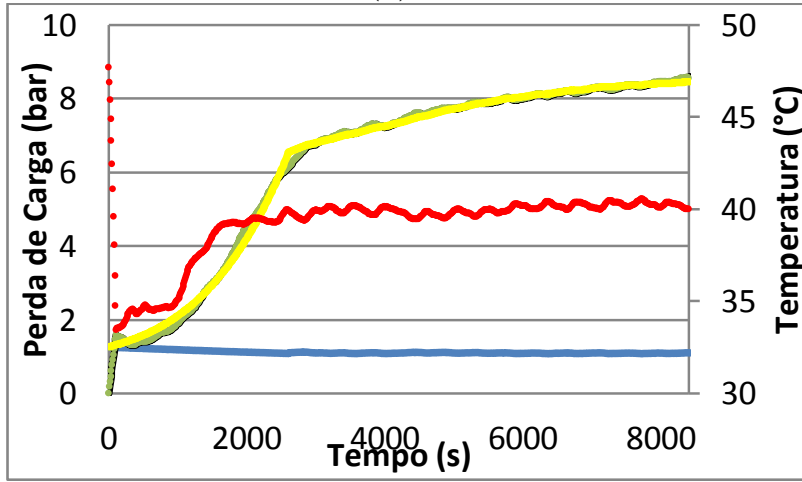

(c)

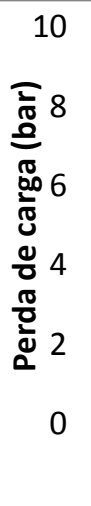

0
50

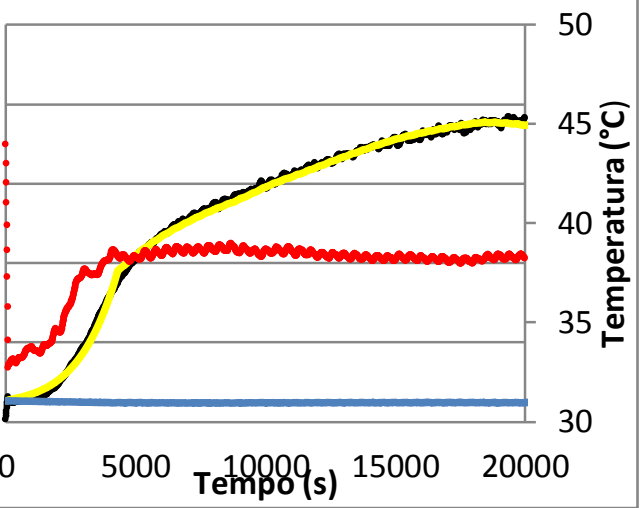

(b)

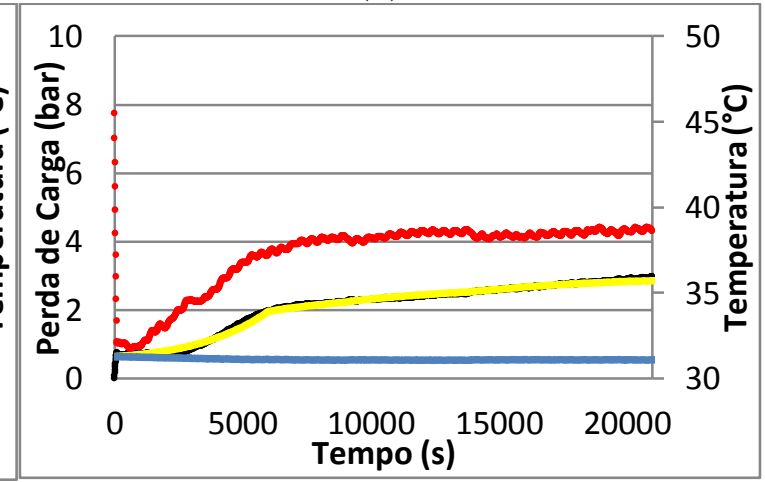

(d)

- Experimental - Modelo —Óleo não parafínico - Tsaída

Figura 3 - Resultados da aplicação do modelo (a) EXP1, (b) EXP2, (c) EXP3, (d) EXP4 
Como pode ser observado, na Figura 3 estão todos os experimentos feitos com o BSW de 5\% (as especificações podem ser observadas na Tabela 1). Analisando-se as figuras, observa-se, em uma primeira etapa, o crescimento da temperatura é acompanhado por um crescimento exponencial da perda de carga. Nos experimentos 2, 3 e 4, a temperatura assume um comportamento constante, seguido por um comportamento mais linear da perda de carga. A exceção do EXP1 que não chegou a apresentar o segundo comportamento, pois o experimento foi interrompido após atingir a pressão de segurança da unidade experimental.

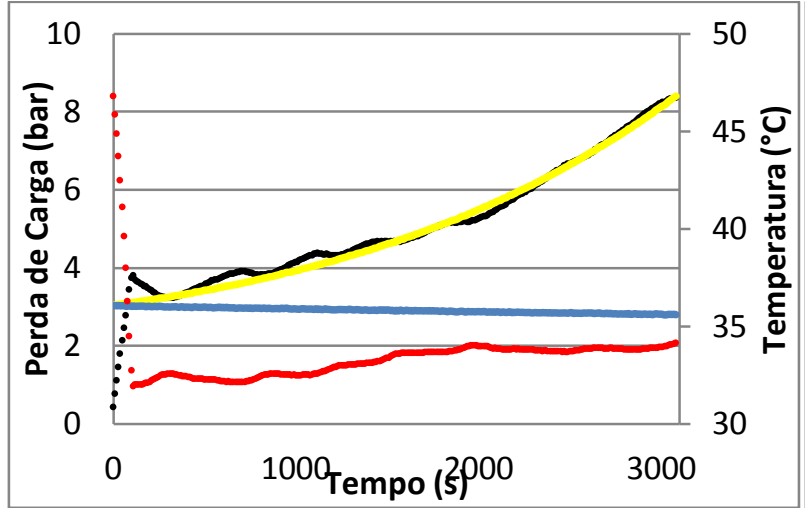

(a)

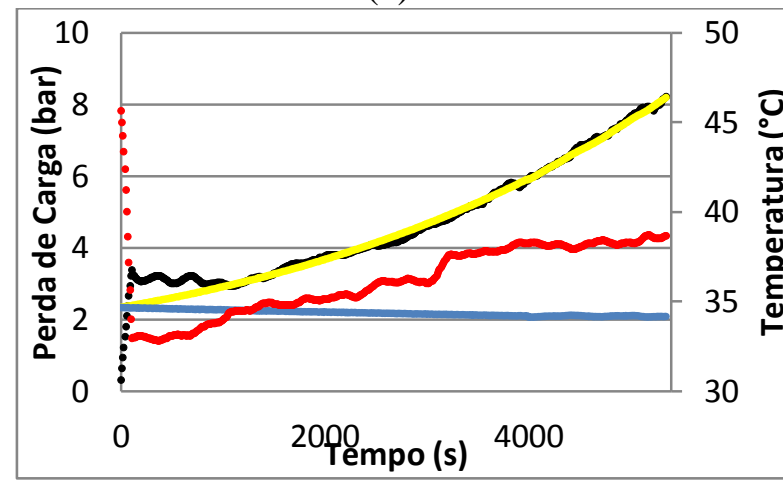

(c)

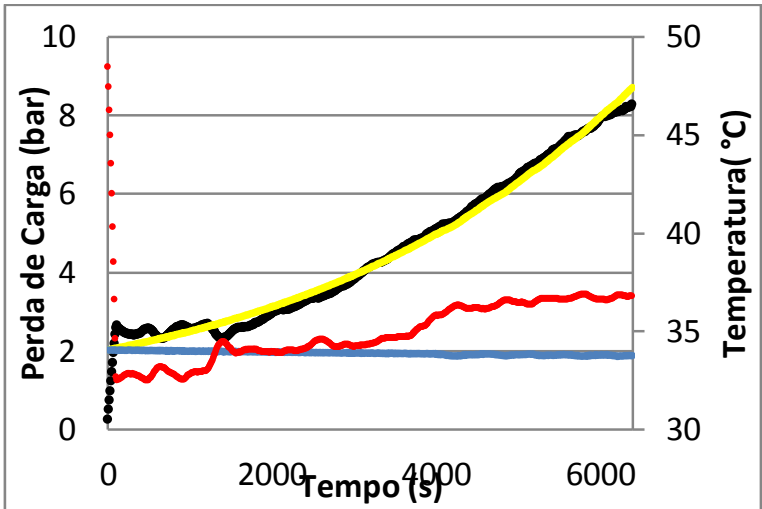

(b)

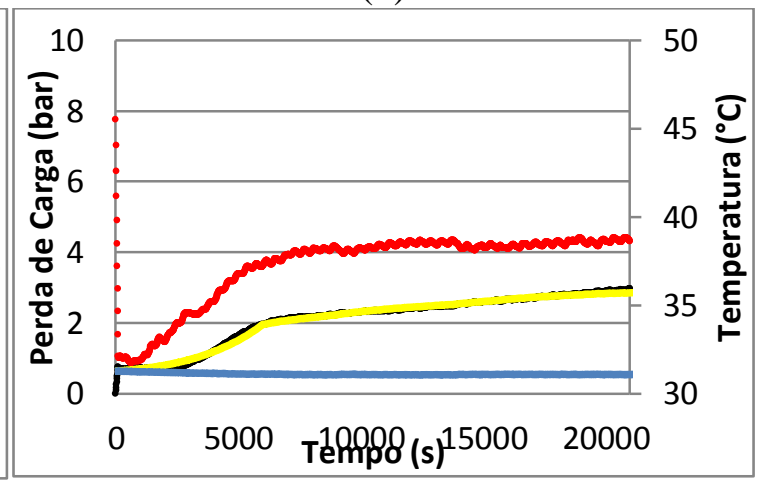

(d)

- Experimental Modelo CÓleo não parafínico - Tsaída

Figura 4 - Resultados da aplicação do modelo (a) EXP5, (b) EXP6, (c) EXP7, (d) EXP8

Agora, comparando-se resultados da Figura 4, onde os experimentos foram realizados com óleos com BSW de 35, com os da Figura 3, é possível perceber que o aumento do teor de água de 5\% para $35 \%$ resulta em um aumento na perda de carga dos escoamentos. Além disso, observa-se também a existência de um período inicial sem variações significativas na perda de carga experimentos, mesmo com o aumento da temperatura do fluido. Este comportamento não pode ser explicado pelo modelo proposto por este trabalho e, por isso, os experimentos com teor de água mais elevados não obtiveram boa representatividade nos períodos iniciais.

A Tabela 2 mostra que o modelo proposto consegue prever adequadamente o comportamento do escoamento do petróleo parafínicos, nas condições de operação relatadas neste trabalho. Também é possível observar que o modelo consegue prever melhor os 
escoamentos mais longos. Isto ocorre devido às limitações do modelo na previsão do comportamento do primeiro período dos dados, caracterizado pelo comportamento exponencial da perda de carga e pelo crescimento da temperatura do fluido. Os experimentos com maior teor de água não foram bem representados pelo modelo nos períodos iniciais, nos quais observa-se um período sem aumento na perda de carga seguido pelo aumento da temperatura do sistema.

Tabela 2-Parâmetro dos ajustes

\begin{tabular}{ccccccccc}
\hline Experimento & EXP1 & EXP2 & EXP3 & EXP4 & EXP5 & EXP6 & EXP7 & EXP8 \\
\hline $\mathrm{R}^{2}$ & 0,996 & 0,998 & 0,998 & 0,995 & 0,992 & 0,991 & 0,983 & 0,998 \\
\hline
\end{tabular}

\section{CONCLUSÃO}

O modelo desenvolvido neste trabalho representou de maneira adequada a perda de carga em escoamento de óleos com precipitação de parafinas. Porém, apesar dos bons resultados obtidos, percebe-se que o modelo pode ser melhorado com o objetivo de explicar melhor o período inicial do escoamento, principalmente quando o fluido possui elevados teores de água.

\section{AGRADECIMENTOS}

Os autores agradecem à Petrobras e ao CNPq.

\section{REFERÊNCIAS}

ABDUVAYT, P., ARIHARA, N., SHARMA, Y., YOSHIDA, Y., A mechanistic model for gas-liquid two-phase flow in slightly inclined pipes: To improve predictions of flow patterns and pressure drop. Journal of the Japan Petroleum Institute. 2002.

COTO, B., MARTOS, C., ESPADA, J. J., ROBUSTILlO, M. D., MERINO-GARCIA, D., PENA, J. L., Study of New Methods to Obtain the n-Paraffin Distribution of Crude Oils and its Application to Flow Assurance. Energy Fuels. v. 25, p. 487-592. 2010.

HAZAN, A. R., KABIR, C. S., SAYARPOUR, M., Simplified two-fase flow modeling in wellbores. Journal of Petroleum Science and Engineering. 2010.

PAULY, J.; DARIDON, J.; E COUTINHO, J. A. P., Solid deposition as a function of temperature in the nC10+ $(\mathbf{n C 2 4}-\mathbf{n C 2 5}-\mathbf{n C 2 6})$ system. Fluid Phase Equilibria. 2004.

SANTOS, J. S. T., FERnANDES, A. C., GIULIETTI, M., Study of paraffin deposit formation using the cold finger methodology for Brazilian crude oils. Journal of Petrolleum science and engineering. v. 45, p. 47-60, 2004.

SHAGAPOV, V. S, MUSAKAEV, N. G., KHABEEV, N. S., BAILEY, S. S.,Methematical modelling of two-phase flow in a vertical well considering paraffin deposits and external heat excenge. International journal of Geat Transfer. v. 47, p. 843-851. 2004. 